\title{
Motivationsarbeit mit Drogenabhängigen: Erfahrungen mit Motivational Interviewing und Case Management
}

Irmgard Vogt

Martin Schmid

Martina Schu

\author{
Motivation Work with Drug Dependent Patients: Experiences with Motivational \\ Interviewing and Case Managment
}

\section{Zusammenfassung}

Dargestellt werden die Erfahrungen, die man bislang in der $\mathrm{Ar}$ beit mit Personen mit Motivational Interviewing (MI) gemacht hat, die illegale Substanzen wie Marihuana, Opiate, Kokain usw. konsumieren oder von ihnen abhängig sind. Unterschieden wird dabei zwischen MI als Add-on-Intervention zur Stärkung der Veränderungsmotivation und MI als Stand-alone-Behandlung, ebenso zwischen verschiedenen Gruppen von Klienten und Klientinnen. Die Ergebnisse zeigen, dass MI in der Mehrzahl der Studien als Add-on-Intervention eingesetzt wird und dass die Erfolge besonders gut sind bei Gruppen von Jugendlichen und Erwachsenen, die relativ gut sozial integriert sind. Bei Gruppen, die sozial bereits stark ausgegrenzt sind, wie zum Beispiel der Klientel, die im bundesdeutschen Modellprojekt zur heroingestützten Behandlung Opiatabhängiger behandelt wird, bewährt sich MI als Interaktionsstil gut in Kombination mit Case Management mit einem hohen Grad an Strukturierung und Vernetzung.

\section{Schliuisselwörter}

Motivational Interviewing · Interaktionsstil • Veränderungsmotivation · Add-on-Intervention oder Stand-alone-Intervention • Case Management

\section{Abstract}

Studies are reviewed in which Motivational Interviewing (MI) was used in working with clients using or abusing illigal drugs such as marijuana, opiates, cocaine etc. The review differentiates between studies, in which MI is an add-on intervention to enhance motivation to change and those in which MI is a standalone therapy, as well as between different groups of clients. In most studies, MI is an add-on intervention, and effects are best with adolescents and adults who are relatively well integrated. In the German heroin-prescription trial, clients are already marginalized. With this group of clients, the combination of MI as style of interaction and case management as structured process with a high potential of networking seems to work very well.

\section{Key words}

Motivational interviewing - interactive style $\cdot$ motivation to change · add-on intervention · stand-alone therapy · case management

\section{Einleitung}

Die Bedeutung der Mitarbeits- oder Veränderungsmotivation von Menschen mit psychischen Störungen allgemein und von Personen, die psychoaktive Substanzen missbrauchen oder von diesen abhängig sind, ist seit langem bekannt (vgl. [1 -4]). Dabei sind zwei Populationen voneinander zu unterscheiden:

Einerseits hat man es mit Menschen zu tun, die ihre Probleme aus eigener Kraft und mithilfe ihrer sozialen Netzwerke lösen können. Allgemein geht man davon aus, dass ca. $80 \%$ aller Men- 
schen, die kürzere oder längere Episoden des Missbrauchs und der Abhängigkeit (nach ICD-10) von einem psychoaktiven Stoff oder auch von mehreren dieser Substanzen durchleben, selbst in der Lage sind, ihr Verhalten zu ändern [5 - 9]. Mit dieser Gruppe wird sich dieser Beitrag nicht beschäftigen, zumal sie gerade nicht die einschlägigen Beratungs- oder Therapieangebote in Anspruch nimmt.

Andererseits gibt es aber auch eine recht große Gruppe von Menschen, die zur Lösung ihrer Substanzprobleme professionelle Hilfe brauchen. Diese Gruppe interessiert im Folgenden und von dieser wiederum diejenigen Frauen und Männer, die illegale Drogen nehmen, sich an sie gewöhnen, von ihnen abhängig werden und die darüber hinaus weitere mit der Abhängigkeit zusammenhängende Probleme entwickelt haben. Der weitaus größte Teil der Studien und Veröffentlichungen, die in den letzten Jahren zum Motivational Interviewing entstanden sind, befasst sich mit Menschen mit Alkoholproblemen und deren Veränderungsmotivation (vgl. den Beitrag von Kremer in diesem Heft). Vergleichsweise wenig ist darüber bekannt, ob und wie sich diese Methode erfolgreich in der Beratung und Behandlung von Menschen einsetzen lässt, die Probleme mit illegalen Drogen haben.

Wer abhängig von illegalen Substanzen ist und seine Selbsthilferessourcen ausgeschöpft hat, kommt eventuell zur Einsicht, dass er oder sie Hilfe von Dritten braucht. Es entsteht also Behandlungsmotivation, die ihn oder sie dazu veranlasst, eine Behandlung aufzusuchen.

Man ging lange Zeit davon aus, dass sich Behandlungsmotivation in einem hohen „Leidensdruck“ manifestiert, also einem subjektiv erlebten Leiden an den eigenen Problemen verbunden mit der subjektiven Einsicht, Hilfe von Dritten zu benötigen, zum Beispiel von SozialarbeiterInnen, PsychologenInnen sowie von ÄrztenInnen, insbesondere von PsychiaterInnen, aber auch von Laienorganisationen wie den Anonymen Alkoholikern bzw. den Narcotics Anonymous usw. Ohne jeden Zweifel gibt es drogenabhängige Frauen und Männer, die ganz erheblich an ihrer Sucht leiden, aber es gibt eben auch viele andere. Dennoch galt „Leidensdruck" lange Zeit als unerlässliche Voraussetzung für die Aufnahme von süchtigen Frauen und Männern in eine Behandlung. Wer wenig oder keinen „Leidensdruck“ hatte, galt als nicht oder noch nicht behandlungsfähig. Unangenehme Aufnahmerituale oder Behandlungsbedingungen galten als Test für die Stärke des „Leidensdrucks“. Wer solche Tests nicht bestand, hatte keine Chance auf Behandlung.

Von diesen Positionen ist man mittlerweile abgerückt. Das liegt einmal daran, dass die Drogenhilfe alles in allem genommen mit diesem Ansatz und dem davon abgeleiteten Vorgehen bei der Behandlungsaufnahme nicht gut gefahren ist (ausführlich in [10, S. 156 ff; vgl. 78, 79, S. 22]). Zum anderen haben sich die Annahmen hinsichtlich Entwicklung und Verlauf von Substanzabhängigkeit verändert mit der Folge, dass man heute auch andere Behandlungsansätze verfolgt [11 - 14].

Dazu hat die Ausformulierung des transtheoretischen Modells der Motivation [15, 16], für Einzelheiten (vgl. Hoyer in diesem Heft) entscheidend beigetragen, das von einem anderen Menschenbild und entsprechend von einem anderen Verständnis der Behandlungs- und Veränderungsmotivation ausgeht. Danach ist Veränderungsmotivation dynamisch und nicht statisch und Beratende können sie systematisch anregen. Sie können die Motivation jedoch auch verringern oder gar zerstören. Es liegt also nicht zuletzt in der Hand der Beratenden, ob Rat Suchende zu einer Behandlung motiviert werden, ob sie bereit sind, sich auf Veränderungen einzulassen und wann sie das in der Entwicklung von Substanzmissbrauch oder Substanzabhängigkeit tun. Daraus haben sich differenzierte Ansätze zur Früh- und Kurzintervention ergeben sowie zur ambulanten und stationären Behandlung.

Wie sich mittlerweile gezeigt hat, eignet sich Motivational Interviewing (MI) ausgezeichnet dazu, die drogenabhängige ${ }^{1}$ Klientel zu ermutigen, eine Behandlung zu beginnen und bis zum regulären Ende hin durchzuhalten. Das belegt eine Reihe von Studien aus der Behandlungsforschung mit Alkohol- und Tabakabhängigen (Übersichtsarbeiten vgl. [17-21]). Auch die vergleichsweise wenigen Studien, die zur Stärkung der Behandlungsmotivation von Drogenabhängigen mit MI vorliegen, sind ermutigend (vgl. [22, 23]). Damit liegen erste evidenzbasierte Einschätzungen über die Effektivität und Wirksamkeit von MI in der Arbeit mit Drogenabhängigen vor, worauf im Folgenden etwas ausführlicher eingegangen werden soll.

\section{MI als Interventionsstrategie zur Förderung der Behandlungsmotivation von Drogenabhängigen allgemein}

MI bzw. eine für die jeweilige Untersuchung adaptierte Form von Motivational Interviewing (Adapted Motivational Interviewing AMI, vgl. [22, 24]) ist in einer Reihe von Therapiestudien vor allem in den USA zum Beispiel mit Opiatabhängigen und Kokainabhängigen eingesetzt worden, um die Behandlungs- und Veränderungsmotivation zu stärken, vor allem die Motivation, den Drogenkonsum zu vermindern bzw. ihn ganz aufzugeben, oder zum Beispiel bei Gruppen, die Methadon als Ersatzdroge erhalten - zur Stabilisierung der Lebensweise insgesamt. Aus Deutschland liegen bislang keine Studien zum Einsatz von Motivational Interviewing in der Beratung und Behandlung von Drogenabhängigen vor. Die Studien aus dem englischen Sprachraum unterscheiden sich in vieler Hinsicht voneinander, wie Auswahl der Klientinnen und Klienten, Art der Störung, Zielsetzung usw. Dennoch findet man bei genauer Betrachtung eine Reihe von Gemeinsamkeiten, die hier kurz dargestellt werden sollen.

(1) In der Mehrzahl der Studien wird AMI gezielt eingesetzt, um die Behandlungs- und Veränderungsmotivation zu fördern (vgl. [25 - 32]). Saunders, Wilkinson und Phillips [33] haben zum Beispiel mit einer Gruppe von Opiatabhängigen, die in ein Methadonprogramm aufgenommen worden sind, eine Kurzintervention nach MI von einer Stunde Dauer durchgeführt. Die Klientinnen und Klienten wurden aufgefordert, positive und negative Aspekte des Opiatkonsums zu beschreiben, ebenso positive und negative Konsequenzen ihrer Abhängigkeit und wie sehr sie dies alles belastet. Auf diese Weise konnten Ambivalenzen im Umgang mit

\footnotetext{
${ }^{1}$ Aus praktischen Gründen verwenden wir den Begriff Drogen als Sammelbezeichnung für illegale psychoaktive Substanzen.
} 
Opiaten bewusst gemacht werden, ebenso Diskrepanzen hinsichtlich der tatsächlichen und der gewünschten Lebensweise. Die Behandler sollten diese Prozesse unterstützen; sie sollten die Klientel jedoch nicht manipulieren oder zu einer Entscheidung überreden. Die Kontrollgruppe bearbeitete ein Informationsheft zur Opiatabhängigkeit, das u.a. Tipps zum Ausstieg aus der Sucht enthielt. Dieser Intervention folgte nach einer Woche eine zweite, ähnlich aufgebaute, die allerdings nur 5 bis 10 Minuten dauerte. Die MI-Gruppe wiederholte noch einmal Ambivalenzen und Diskrepanzen in ihrem Umgang mit Drogen, die Kontrollgruppe die Informationen zu den Stoffen. 3 und 6 Monate nach den Interventionen folgten weitere Nachuntersuchungen, um abzuklären, ob sich kurz-, mittel- und langfristige Unterschiede in der Veränderungsmotivation der beiden Gruppen feststellen lassen. In dieser wie in einer Reihe weiterer Studien konnte man feststellen, dass Kurzinterventionen mit MI die Behandlungs- und Veränderungsmotivation positiv beeinflussen, dass die Klientinnen und Klienten also stärker motiviert sind, an der Behandlung teilzunehmen, und dass sie mehr Interesse daran zeigen, ihren Drogenkonsum zu kontrollieren bzw. zu verringern.

Variationen ergeben sich hinsichtlich der Dauer der Kurzintervention, die sich von wenigen Minuten bis auf vier Stunden, verteilt über mehrere Sitzungen, hinziehen kann. Variationen findet man auch im Hinblick auf die Art, in der AMI eingesetzt wird, und auf die Themen, die besonders betont werden. Jedoch geht es immer darum, den MI-typischen Interventionsstil beizubehalten, der getragen wird von Empathie, einfühlendem Verstehen, Akzeptanz und der Stärkung der Kompetenzerwartungen bzw. der Selbstwirksamkeit der Klientinnen und Klienten.

Lovejoy u. Mitarb. [34] konnten in einer qualitativen Studie zeigen, dass viele Klienten und Klientinnen, die die Behandlung mit einem hohen Maß an Misstrauen gegenüber den Behandlern begonnen hatten, ihre Einstellung nachhaltig geändert haben. Aus Misstrauen wurde Vertrauen, aus Hoffnungslosigkeit Hoffnung auf Veränderung, auf eine weitere Chance im Leben.

(2) In der Regel folgen auf die Kurzinterventionen weitere therapeutische Interventionen, wobei besonders häufig kognitive verhaltenstherapeutische Verfahren (KVT) eingesetzt werden (vgl. [35]).

(3) In den meisten Studien findet die AMI-Intervention als Einzelfallberatung statt, in einigen wenigen jedoch in (kleinen) Gruppen [36, 37]. Haben Klienten und Klientinnen erst einmal Vertrauen zu ihren Behandlern aufgebaut, schätzen sie die Arbeit in Gruppen. In der Kommunikation mit anderen erfahren sie, dass diese ähnliche Probleme haben wie sie selbst und ähnliche Schwierigkeiten, wenn es darum geht, Veränderungen umzusetzen [34].

(4) Die Weiterbehandlung der Klientinnen und Klienten erfolgt fast immer in der Gruppe. Das liegt nicht zuletzt daran, dass die amerikanische Drogenhilfe Einzelfallbetreuung kaum kennt.

(5) In den Kontrollgruppen wird sehr häufig mit psychoedukativen Informationen und Materialien gearbeitet. Die Klientinnen und Klienten erhalten Studienhefte, die zum Selbststudium anregen sollen und die sie entweder allein oder in der Gruppe durcharbeiten.
Die Ergebnisse dieser Studien, in denen es in erster Linie darum geht, die Behandlungs- und Veränderungsmotivation der Klientel zu stärken und zu unterstützen, sind gemischt. Einige Studien belegen, dass die Kurzinterventionen mit AMI unabhängig vom Setting effektiv sind. AMI stärkt demnach die Motivation, eine Behandlung anzufangen und durchzuhalten, sowohl dann, wenn die Intervention auf den Einzelfall abgestellt ist, als auch dann, wenn sie in (kleinen) Gruppen stattfindet. Was das Setting betrifft, ist aber noch eine Reihe von Fragen offen, die in weiteren Studien zu klären sind, u. a. diejenige, welche Gruppen von Klientinnen und Klienten mehr von AMI profitieren, wenn diese auf den Einzelfall zugeschnitten ist oder wenn sie als Gruppenintervention implementiert wird. Wie wichtig der Erfahrungsaustausch in der Gruppe für Drogenabhängige sein kann, belegen die Ergebnisse von Lovejoy u. Mitarb. [34] nachdrücklich.

Andere Studien kommen zu anderen Ergebnissen. Danach ist MI/ AMI nicht effektiver als andere Interventionsmethoden, sondern bestenfalls ebenso gut [30,32]. Das liegt wohl einerseits daran, dass es zwischen den verschiedenen Studien erhebliche methodische Unterschiede gibt hinsichtlich der Umsetzung von MI bzw. AMI. Zum anderen unterscheiden sich die Stichproben, die behandelt wurden, sehr stark voneinander. Klientinnen und Klienten, die unter sehr vielen negativen Folgen ihres Drogenkonsums leiden, sind offenbar viel weniger leicht zu einer Behandlung und zu den damit verbundenen Verhaltensänderungen zu motivieren $[30,38,39]$ als Personen, die gerade erst am Anfang einer Drogenkarriere stehen. Für Drogenabhängige mit langen Karrieren, von denen viele schon eine Reihe von Ausstiegsversuchen hinter sich haben und die von den Versprechungen der Behandler oft enttäuscht wurden [40], bietet es sich an, MI mit anderen Interventionsverfahren zu verbinden, zum Beispiel mit Case Management, was zu einer stärkeren Strukturierung der Behandlung beiträgt ebenso wie zu einer Optimierung der Vernetzung mit anderen helfenden Institutionen [41-43]. Ein gut strukturierter Behandlungsablauf sowie intensive Vernetzung mit anderen Hilfen sind für diese Klientel offenbar dann besonders vorteilhaft, wenn die Beziehung zum Case Manager gut ist, und das ist vor allem dann der Fall, wenn der Interaktionsstil an MI/AMI orientiert ist.

MII als Interventionsstrategie mit speziellen Subgruppen von Drogenabhängigen

In den letzten Jahren ist die Diskussion über die Bedeutung von Mehrfachdiagnosen bei Drogenabhängigen stark in den Vordergrund gerückt. Man diskutiert allenthalben darüber, welche Verfahren zur Behandlung von Drogenabhängigen, bei denen weitere psychische Störungen diagnostiziert worden sind - so genannte Doppeldiagnosen (DD) -, geeignet sind und welche nicht $[44,45]$.

Eine Reihe von Studien zeigt, dass AMI die Bereitschaft von Patientinnen und Patienten mit Doppeldiagnosen, nach einer stationären Behandlung an einer ambulanten teilzunehmen, erhöht [44, 46-51]. AMI in Kombination mit KVT stärkt die Veränderungsmotivation hinsichtlich des Drogenkonsums: Patienten und Patientinnen finden sich eher bereit, diesen zu reduzieren oder ganz aufzugeben $[52,53]$. Auch scheint AMI sich zumindest 
kurzfristig zu bewähren, wenn es um eine Erhöhung der Compliance bei der Medikamenteneinnahme, zum Beispiel bei drogenabhängigen psychotischen Patienten, geht $[54,55]$. Alle hier erwähnten Studien arbeiten mit Interventionen, die für den Einzelfall zugeschnitten sind. Gruppenprogramme sind bislang nicht ausgearbeitet und erprobt worden.

Besondere Umstände bestimmen auch die Behandlung von Abhängigen, die unfreiwillig in die Behandlung kommen oder die im Gefängnis sitzen und denen dort eine solche angeboten wird. Es liegt auf der Hand, dass MI und AMI nicht für Personen entworfen worden sind, die keinerlei eigene Motivation zu einer Behandlung zeigen. Dennoch spricht einiges dafür, AMI auch mit diesen Gruppen auszuprobieren: Donovan und Rosengreen [6] weisen nachdrücklich darauf hin, dass Personen, die unfreiwillig eine Behandlung aufnehmen, durchaus Veränderungsmotivation entwickeln und sich auf Verhaltensänderungen einlassen. Evaluationsstudien belegen zudem, dass man in manchen Gruppen der unfreiwillig Behandelten mehr Personen findet, die ihren Substanzkonsum dauerhaft reduziert haben oder die ganz abstinent sind, als in Gruppen von Personen, die sich freiwillig in Behandlung begeben haben.

Bisher sind die Ergebnisse der Interventionen mit MI oder AMI mit drogenabhängigen Straffälligen wenig spektakulär. Vergleicht man MI und AMI mit anderen Ansätzen, dann findet man keinen oder nur einen sehr geringen Effekt [56]. Da auch andere Studien zeigen, dass AMI nicht in jedem Fall einer konfrontativen Intervention überlegen ist [32], ist zu fragen, ob für Strafgefangene und vor allem für die Gewalttäter unter ihnen andere Behandlungsansätze besser geeignet sind, Verhaltensänderungen einzuleiten.

\section{MI als Interventionsstrategie mit Jugendlichen}

MI und AMI sind gut erprobte Interventionsansätze mit Jugendlichen und jungen Erwachsenen, insbesondere mit College-Studenten, die Probleme mit ihrem Alkoholkonsum haben (vgl. [57-59]. Dagegen sind MI und AMI bislang nur selten eingesetzt worden bei jungen Menschen mit Drogenproblemen. Immerhin konnte Dennis [60] zeigen, dass man mit MI jugendliche Marihuana-Raucher erreichen kann und dass die Intervention in Kombination mit KVT sehr erfolgreich ist. Besonders effektiv waren kurze Interventionssequenzen mit zwei MI-Modulen und drei KVT-Modulen, die als Gruppenprogramm durchgeführt wurden. Das Ergebnis ist bemerkenswert, da man weiß, dass Jugendliche, die Drogen konsumieren, therapeutisch ohnehin schwer zu erreichen sind [61] und sich einer Auseinandersetzung mit diesen Verhaltensweisen gerne entziehen. Auch die Studie von Aubrey [62] belegt den positiven Effekt von AMI bei Jugendlichen hinsichtlich der Behandlungs- und Veränderungsmotivation.

Diese ersten Ergebnisse mit MI und AMI mit Jugendlichen ermutigen dazu, die Verfahren auch für die Prävention zu nutzen bzw. für Gruppen von jungen Menschen, die mit Drogen experimentieren, aber noch keine festen Konsumgewohnheiten entwickelt haben. Es liegt nahe, MI und AMI vor allem zur Beratung der ansteigenden Zahl von jungen Menschen einzusetzen, die Cannabis konsumieren [72 - 74] und sich offensichtlich der Probleme, die damit ver- bunden sind, nicht bewusst sind. Man würde damit Neuland im Umgang von jungen Menschen beschreiten, die zwar schon über mehr oder weniger intensive Erfahrungen mit Drogen verfügen, aber noch nicht abhängig sind. Das setzt aber voraus, dass die Drogenberatungsstellen bzw. die Einrichtungen der Jugendhilfe gezielt BeraterInnen abstellen, die in MI/AMI geschult sind und die sich auf die Arbeit mit jugendlichen KonsumentenInnen von Cannabis (und Ecstasy) spezialisiert haben. Wenngleich in Deutschland erste Ansätze in diese Richtung entstehen [75, 76], kann doch von einer entsprechenden Umorganisation von Drogenbzw. Jugendhilfe (noch) nicht gesprochen werden.

\section{MI/AMI als Behandllungsverfahren}

Wie die Durchsicht der einschlägigen Literatur zeigt, werden MI und AMI vornehmlich als Kurzinterventionen eingesetzt mit dem Ziel, die Behandlungs- und die Veränderungsmotivation zu befördern oder aufrechtzuerhalten. Man findet nur wenige Studien zur Behandlung von Drogenproblemen, die über diese Zielsetzung hinausgehen und in denen der Ansatz für die ganze Dauer der Behandlung durchgehalten wird. Stephens u. Mitarb. [24, 63] orientierten sich in ihrer Studie bei der Behandlung von erwachsenen Marihuanakonsumenten am Vorgehen des Drinker's Check-Up [64, 65] und übersetzten die dort eingesetzten Verfahren auf ihre Gruppe. Die Behandlung mit AMI beschränkte sich auf zwei Sitzungen von jeweils ca. 90 Minuten sowie einer schriftlichen Intervention zwischen der ersten und der zweiten Sitzung. Um die Wirksamkeit der Intervention zu prüfen, wurde eine Vergleichsgruppe untersucht mit insgesamt 12 Gruppensitzungen von jeweils 2 Stunden Dauer, in der u.a. mit Methoden der Rückfallprävention gearbeitet wurde. Darüber hinaus wurde eine nicht behandelte Gruppe untersucht, um Plazebo-Effekte zu kontrollieren. Die Ergebnisse zeigen, dass sowohl in der Untersuchungsgruppe als auch in der Vergleichsgruppe der Marihuanakonsum signifikant zurückgeht und dass dieser Effekt bei beiden Interventionsverfahren zu beobachten ist. Bedenkt man, dass die Intervention mit AMI weniger zeitintensiv ist als die Arbeit mit der Vergleichsgruppe, dann spricht das für die Überlegenheit dieses Verfahrens. Berücksichtigt man hier auch die einschlägigen Ergebnisse der Behandlung von zum Beispiel Studierenden mit Alkoholproblemen mit MI/AMI, dann gibt es gute Gründe, davon auszugehen, dass sich das Verfahren als „Standalone“-Intervention bei einer bestimmten Klientel bewährt. Besonders erfolgreich ist es bei Klientinnen und Klienten, die relativ gut ausgebildet sind und die relativ gut funktionierende soziale Netzwerke haben.

Betrachtet man hingegen die typische deutsche Klientel von Einrichtungen der ambulanten und stationären Drogenhilfe [66], dann zeigt sich schnell, dass diese Gruppe nur für eine Minderheit der Klientinnen und Klienten steht: Die meisten Menschen, die die Einrichtungen der Drogenhilfe aufsuchen, zeichnen sich gerade durch schlechte Schul- und Berufsausbildung, fehlende Integration in den Arbeitsmarkt, hohe soziale Desintegration und das Fehlen von sozialen Netzwerken (abgesehen von der Drogenszene und der Drogenhilfe) aus. Fasst man die vorliegenden Studien und Auswertungen von Klientendokumentationssystemen zusammen, so trifft man in den Einrichtungen der Drogenhilfe gehäuft auf Klientinnen und Klienten mit lang 
andauernden Drogenkarrieren, vielfachen und komplizierten Problemlagen, wenig Ressourcen, beschädigtem Selbstwertgefühl und minimaler Selbstwirksamkeitserwartung. Kurzum: Die durchschnittliche Klientel deutscher Drogenhilfeeinrichtungen unterscheidet sich dramatisch von der Klientel, die in der Mehrzahl der hier berücksichtigten amerikanischen Studien untersucht worden ist, und es erscheint eher zweifelhaft, dass den deutschen Klientinnen und Klienten mit wenigen Kurzinterventionen tatsächlich geholfen werden kann. Selbst wenn es gelingen sollte, die Veränderungsmotivation durch Motivational Interviewing massiv zu unterstützen, bleibt die Frage offen, wo bei einer derart marginalisierten und demoralisierten Klientel die Veränderungskompetenz herkommen soll.

Aus diesen Gründen hat man sich im bundesdeutschen Modellprojekt zur heroingestützten Behandlung von Opiatabhängigen für die Kombination von MI/AMI mit Case Management entschieden. Opiatabhängige, die den Zielgruppendefinitionen und Einschlusskriterien der Heroinstudie entsprechen - MethadonSubstituierte, die nur unzureichend von der Substitutionsbehandlung profitieren, sowie Drogenabhängige, die in den letzten Monaten nicht von der Drogenhilfe erreicht worden sind, mit mehrjährigen Abhängigkeitsverläufen, hohem Konsum und gesundheitlichen Beeinträchtigungen -, sind gerade nicht KonsumentInnen in einem frühen Stadium ihrer Suchtkarriere und es handelt sich auch nicht um Personen mit hohem Selbsthilfepotenzial und funktionierenden sozialen Netzwerken, sondern KlientInnen mit komplexen Problemlagen, wenig Ressourcen und starken gesundheitlichen Belastungen einschließlich von Doppeldiagnosen. Motivational Interviewing steht für die Grundhaltung der BeraterInnen und für die Technik der Gesprächsführung, Case Management für ein strukturiertes und kontrolliertes sozialarbeiterisches Vorgehen mit Hilfeplanung, Hilfeerschließung und Hilfeleistung. Auf diesen Ansatz soll abschließend noch kurz eingegangen werden.

\section{MI in Kombination mit Case Management zur Behandlung} von chronisch Opiatabhängigen

Case Management in der Drogenhilfe [77] versteht sich als eine Weiterentwicklung der Einzelfallhilfe, die besonderes Gewicht auf eine strukturierte Vorgehensweise, kontinuierliche und nachhaltige Hilfeleistung sowie den Aufbau und die Organisation individueller Unterstützungsnetzwerke legt. Vorausgegangene Studien [42] haben gezeigt, dass Case Management prinzipiell geeignet ist, Menschen mit langjährigen Suchtkarrieren Hilfen zu erschließen. Dabei wurde aber auch deutlich, dass Case Management durch eine gezielte Bearbeitung motivationaler Prozesse optimiert werden sollte, in diesem Fall also durch MI. Für die Heroinstudie wurde in enger Anlehnung an die Prinzipien von MI ein Manual erarbeitet, das die Verbindung der beiden Ansätze erläutert [41]. Die in der Studie eingesetzten Case Manager, in der Regel ausgebildete SozialarbeiterInnen oder SozialpädagogInnen mit Erfahrung in der Suchtarbeit, werden auf dieser Grundlage ausführlich geschult. Der Schulungsumfang liegt bei ca. 12 Tagen und umfasst neben einem Basistraining in Motivational Interviewing und Case Management auch zeitlich versetzte Auffrischungskurse. Schwerpunkte der Schulung sind - neben dem Case-Management-Verfahren - die Grundprinzipien und wichtigsten Methoden von MI mit:

- empathischer Grundhaltung

- Entwickeln von Ambivalenzen und Diskrepanzen

- Arbeiten mit dem Widerstand

- Förderung und Unterstützung der Kompetenz- oder Selbstwirksamkeitserwartung.

Halbjährliche methodenzentrierte Coaching-Termine dienen der Sicherung der fachlichen Qualität.

Die bisherigen Erfahrungen mit Manualisierung, Schulung und Coaching zeigen, dass die psychosozialen Fachkräfte die angebotenen Methoden positiv aufnehmen und zuversichtlich sind, mit der Kombination aus MI und Case Management ihren Klientinnen und Klienten effektiv helfen zu können. Deutlich wurde aber auch, dass manualisiertes Arbeiten für die psychosoziale Drogenhilfe in Deutschland noch neu und ungewohnt ist.

Methodisch geht es auch bei der Kombination der beiden Ansätze darum, offene Fragen zu stellen, aktiv und reflexiv zuzuhören, Aussagen der Klienten und Klientinnen zusammenzufassen, Rückmeldungen und positive Bestätigungen zu geben und selbstmotivierende Aussagen, die Veränderungsabsichten transportieren, hervorzurufen und zu unterstützen. Die Grundprinzipien und die Grundhaltung von MI und das damit verbundene methodische Vorgehen sind zu verbinden mit dem Ablaufschema von Case Management mit Assessment und Problem- und Ressourcenanalyse, Hilfeplanung und Zielbestimmung, Durchführung und Monitoring, Re-Assessment, Beendigung und Evaluation der Behandlung.

Die Kontaktaufnahme zwischen Case Manager und KlientIn fokussiert auf den Aufbau einer guten Beziehung, die getragen wird von Empathie, Akzeptanz und Respekt. Die Klientel steht im Mittelpunkt der Begegnung; der Klient bzw. die Klientin bestimmt die Themen, über die zunächst gesprochen wird. Im Verlauf dieser Begegnung erläutert der Case Manager seine Rolle und seine Arbeitsweise und bemüht sich darum, den Klienten oder die Klientin zur weiteren Zusammenarbeit zu motivieren.

Das Assessment zielt auf einen umfassenden Einblick in die Lebenssituation der Klientin bzw. des Klienten und auf eine Klärung der Problemlagen und stellt die erste Hürde dar im Hinblick auf die Verbindung der beiden Ansätze. Der Case Manager soll die Klientin oder den Klienten - unter Nutzung der Methoden von MI - dazu ermutigen, über sich selbst zu sprechen und ihm Informationen zu einer Reihe von Lebensbereichen zu geben. Wie die Praxis zeigt, funktioniert das dann sehr gut, wenn der Aufbau der Beziehung in der ersten Begegnung gelungen ist. Dann helfen in der Phase des Assessments die MI-typischen Haltungen und Methoden, nicht nur Informationen über Probleme und Ressourcen zu sammeln, sondern auch die subjektive Bedeutung der jeweiligen Handlungen und Lebensweisen zu verstehen. Für den Case Manager liegt eine der Schwierigkeiten in dieser Phase darin, immer wieder die Balance zwischen den MItypischen Haltungen und Methoden und den Handlungsanforderungen, die mit dem Case-Management-Verfahren assoziiert sind, zu finden. 
Die Informationen, die im Assessment gewonnen werden, liefern die Grundlage für die Problemanalyse sowie die Zieldiskussion und den Hilfe- und Veränderungsplan. Wie Schu et al. [67] schreiben, gilt der Hilfeplan als das Prinzip aller Arbeitsschritte von Case Management. Im Hilfeplan wird das weitere Vorgehen festgelegt, ebenso die Vernetzung mit anderen formalen Hilfeeinrichtungen und mit informellen Unterstützungsnetzwerken. Offenbar ist es aber nicht einfach, gewohnte Arbeitsroutinen zu verändern, Ziele zu definieren und prospektiv einen Hilfeplan zu erarbeiten. Zudem verwickeln opiatabhängige KlientInnen ihre Case Manager immer von neuem in Kriseninterventionen, die Soforthilfen erfordern und insofern in gewisser Weise langfristige Planungen erschweren. Es wird eine Aufgabe der Evaluation sein, herauszuarbeiten, in welchen Fällen und unter welchen Bedingungen es gelingt, Hilfepläne zu erstellen und umzusetzen. Kommt es nicht zur Erarbeitung eines Hilfeplans, ist genauer zu analysieren, welche Prozesse dem entgegenstehen. Auf die Ergebnisse dieser Analysen kann man gespannt sein.

An die Phase der Hilfeplanung schließen sich Umsetzung und Durchführung der verabredeten Schritte an. In dieser Phase liegt ein Schwerpunkt der Arbeit der Case Manager in der Organisation des Hilfenetzwerkes und im Monitoring des Verlaufs. Kontinuierlich im Rahmen des Monitorings und zusätzlich zu bestimmten Zeitpunkten durch ein Re-Assessment soll überprüft werden, ob die verabredeten Ziele und Umsetzungsschritte erreicht werden konnten oder nicht. Darauf aufbauend, wird die Hilfeplanung fortgeschrieben.

Fasst man die bisherigen Erfahrungen von MI und Case Management in der Arbeit mit chronisch Opiatabhängigen zusammen, dann ist festzuhalten, dass sich beide Verfahren sehr gut miteinander kombinieren lassen und dass sie sich in der Praxis bewähren. Zentrale Aufgabe der Case Manager ist es, die einzelnen Schritte im Rahmen des Case Managements an die Stadien der Veränderungsmotivation der Klientinnen und Klienten anzupassen bzw. mit dem Interaktionsstil und den Methoden von MI die Veränderungsmotivation jeweils so zu stärken, dass Case Management möglich wird. Werden die im Rahmen der Hilfeplanung verabredeten Ziele nicht erreicht, so liegt dies meist daran, dass diese nicht adäquat und zu wenig an die Veränderungsmotivation und -kompetenz des jeweiligen Klienten angepasst waren.

\section{Ausblick}

Das große Interesse, das die Drogenhilfe auch in Deutschland zurzeit an MI zeigt, steht bislang in einem unproportionalen Verhältnis zu gesicherten Erkenntnissen hinsichtlich der Anwendbarkeit und Wirksamkeit von MI insbesondere bei langjährig Drogenabhängigen mit komplexen Problemlagen. Dennoch ist dieses Interesse verständlich, bietet sich doch der Drogenhilfe die Chance, ein besseres Verständnis von Veränderungsbereitschaft zu entwickeln und sich Verfahren zur Unterstützung von Veränderungsmotivation anzueignen, die sich zudem gut mit anderen Methoden kombinieren lassen.

Eine besondere Herausforderung ist MI für niedrigschwellige Einrichtungen wie Kontaktläden, Krisenzentren und Konsumräume: Erst zaghaft wird versucht, MI-basierte Kurzinterventio- nen auch in diesen Settings einzusetzen, was auch heißt, dem Begriff „Akzeptanz“2 eine neue Bedeutung zu geben.

Mit der zunehmenden Zahl der (kontrollierten) Studien mit Drogenabhängigen, in denen MI/AMI allein oder in Kombination mit anderen Verfahren eingesetzt wird, nehmen die Belege zu, die die Effektivität und Wirksamkeit dieses Interventionsverfahrens unterstreichen. MI/AMI stärkt nicht nur die Behandlungs- und Veränderungsmotivation von Personen, die noch am Anfang einer Drogenkarriere stehen, sondern auch von jenen, die bereits (chronisch) abhängig sind. Allerdings sollte man keine Wunder erwarten; MI/AMI kann nicht alle Drogenabhängigen erreichen und es kann nicht alle dazu motivieren, ihre Lebensweisen zu ändern. Im Vergleich zu vielen anderen Methoden verspricht MI/ AMI jedoch einen besseren Zugang zur Klientel und eine vergleichsweise größere Effektivität hinsichtlich der Behandlungsund Veränderungsmotivation.

Voraussetzung dafür ist eine intensive Schulung der BeraterInnen in MI/AMI. Das ist deshalb unerlässlich, weil Miller et al. $[70,71]$ nachgewiesen haben, dass die Fähigkeiten der Beratenden entscheidend für die (erfolgreiche) Umsetzung des Ansatzes sind. Davon hängt es nämlich ab, ob sie ihre Klientel erreichen, wie gut oder schlecht ihnen das gelingt, wie positiv oder negativ sie deren Behandlungs- und Veränderungsmotivation beeinflussen und $\mathrm{zu}$ welchen Verhaltensänderungen es schließlich kommt. So gesehen stellt MI/AMI in erster Linie eine Herausforderung für die Beratenden dar.

\section{Literatur}

${ }^{1}$ Brenk-Schulte E, Pfeiffer W. Therapiemotivation in der Behandlung des Alkoholismus. München: Röttger, 1987

2 Petry J. Behandlungsmotivation. Grundlagen und Anwendungen in der Suchttherapie. Weinheim: Beltz, 1993

${ }^{3}$ Fleischmann H, Klein HE (Hrsg). Behandlungsmotivation, Motivationsbehandlung. Suchtkranke im Psychiatrischen Krankenhaus. Freiburg: Lambertus, 1995

${ }^{4}$ Veith A. Therapiemotivation. Zur Spezifizierung einer unspezifischen Therapievariablen. Opladen: Westdeutscher Verlag, 1997

${ }^{5}$ Tucker JA, King MP. Resolving alcohol and drug problems: Influences of addictive behavior change and help-seeking processes. In: Tucker JA, Donovan DM, Marlatt GA (Hrsg). Changing addictive behavior. New York: Guilford Press, 1999; $97-126$

${ }^{6}$ Donovan DM, Rosengreen DB. Motivation for behavior change and treatment among substance abusers. In: Tucker JA, Donovan DM, Marlatt, GA (Hrsg). Changing addictive behavior. New York: Guilford Press, 1999; $127-159$

${ }^{7}$ Miller WR. Enhancing motivation for change. In: Miller WR, Heather N (Hrsg). Treating addictive behaviors. New York: Plenum Press, 1998; 2. Auflage: $121-132$

${ }^{8}$ Happel HV, Fischer R, Wittfeld I. Selbstorganisierter Ausstieg. Überwindung der Drogenabhängigkeit ohne professionelle Hilfe. Endbericht. Frankfurt: FH Frankfurt (Typoskript),

${ }^{9}$ Klingemann H. Spontanverläufe unbehandelter Suchtkrankheit. In: Uchtenhagen A, Zieglgänsberger W (Hrsg). Suchtmedizin. Konzepte, Strategien und therapeutisches Management. München: Urban \& Fischer, 2000; 406-410

${ }^{10}$ Schmid M. Drogenhilfe in Deutschland. Entstehung und Entwicklung 1970-2000. Frankfurt am Main: Campus, 2003

2 „Akzeptanz“ dient als Schlüsselbegriff für eine ganze Richtung der Drogenhilfe, jedoch ohne jede Anleihe oder Auseinandersetzung mit dem von Rogers [68] in die Therapie eingeführten Akzeptanzbegriff [69]. 
${ }^{11}$ Miller WR, Rollnick S. Motivational interviewing. Preparing people to change addictive behavior. New York: Guilford Press, 1991

${ }^{12}$ Miller WR, Rollnick S. Motivational interviewing: Preparing people for change. 2. Auflage. New York: Guilford Press, 2002

${ }^{13}$ Miller WR. Enhancing motivation for change in substance abuse treatment. Treatment Improvement Protocol (TIP) Series 35. Rockville: Center for Substance Abuse Treatment, 1999

${ }^{14}$ Marlatt GA, Gordon JR (Hrsg). Relapse prevention: Maintenance strategies in the treatment of addictive behaviors. New York: Guilford Press, 1985

15 Prochaska JO, DiClemente CC. Transtheoretical therapy: Toward a more integrative model of change. Psychotherapy. Theory, Research and Practice 1982; 19: 276-288

${ }^{16}$ Prochaska JO, DiClemente CC. The transtheoretical approach: Crossing traditional boundaries of change. Homewood: Dorsey Press, 1984

17 John U, Hapke U, Rumpf HJ et al. Prävalenz und Sekundärprävention von Alkoholmissbrauch und -abhängigkeit in der medizinischen Versorgung. Baden-Baden: Nomos, 1996

${ }^{18}$ John U, Veltrup C, Driessen M et al. Motivationsarbeit mit Abhängigen. Freiburg: Lambertus, 2000

${ }^{19}$ Pittrich W, Rometsch W, Sarrazin D (Hrsg). Kurzintervention und motivierende Gesprächsführung. Münster: Landschaftsverband Westfalen-Lippe, 2000

${ }^{20}$ Miller WR, Brown JM, Simpson TL et al. What works? A methodological analysis of the alcohol treatment outcome literature. In: Hester, RK, Miller WR (Hrsg). Handbook of alcoholism treatment approaches: Effective alternatives. Boston: Allyn \& Bacon, 1995; 2. Auflage: 12-44

${ }^{21}$ Noonan WC, Moyers TB. Motivational interviewing: A review. Journal of Substance Misuse 1997; 2: 8-16

${ }^{22}$ Dunn C, DeRoo L, Rivara FP. The use of brief interventions adapted for motivational interviewing across behavioral domains: A systematic review. Addiction 2001; 96: 1725 - 1742

${ }^{23}$ Zweben A, Zuckoff A. Motivational interviewing and treatment adherence. In: Miller WR, Rollnick S (Hrsg). Motivational interviewing. Preparing people for change. 2. Auflage. New York: Guilford Press, 2002; 299-319

24 Burke BL, Arkowitz H, Dunn C. The efficacy of motivational interviewing. In: Miller WR, Rollnick S (Hrsg). Motivational interviewing. Preparing people for change. 2. Auflage. New York: Guilford Press, 2002; 217-250

${ }^{25}$ Baker A, Heather N, Wodak A et al. Evaluation of a cognitive-behavioral intervention for HIV prevention among injecting drug users. AIDS 1993; 7: $247-256$

${ }^{26}$ Baker A, Kochan N, Dixon J et al. Controlled evaluation of a brief intervention for HIV prevention among injecting drug users not in treatment. AIDS Care 1994; 6: 559-570

${ }^{27}$ Baker A, Boggs TG, Lewin TJ. Randomized controlled trial of brief cognitive-behavioural interventions among regular users of amphetamines. Addiction 2001; 96: 1279-1287

${ }^{28}$ Booth RE, Kwiatkowski C, Iguchi MY et al. Facilitating treatment entry among out-of-treatment injection drug users. Public Health Reports 1998; 113 (Suppl. 1): 116 - 128

${ }^{29}$ Stotts AL, Schmitz JM, Rhoades HM et al. Motivational interviewing with cocaine-dependent patients: A pilot study. Journal of Consulting and Clinical Psychology 2001; 69: 858-862

${ }^{30}$ Donovan DM, Rosengreen DB, Downey L et al. Attrition prevention with individuals awaiting publicly funded drug treatment. Addiction 2001; 96: $1149-1160$

31 Saunders B, Wilkinson C, Allsop S. Motivational intervention with heroin users attending a methadone clinic. In: Miller WR, Rollnick $S$ (Hrsg). Motivational interviewing. Preparing people to change addictive behavior. New York: Guilford Press, 1991; 279-292

${ }^{32}$ Schneider RJ, Casey J, Kohn R. Motivational versus confrontational interviewing: A comparison of substance abuse assessment practices at employee assistance programs. Journal of Behavioral Health Services \& Research 2000; 27: 60-74

33 Saunders B, Wilkinson C, Phillips M. The impact of brief motivational intervention with opiate users attending a methadone programme Addiction 1995; 90: 415-424

${ }^{34}$ Lovejoy M, Rosenblum A, Magura S et al. Patients perspective on the process of change in substance abuse treatment. Journal of Substance Abuse Treatment 1995; 12: 269-282

${ }^{35}$ Beck AT, Wright FD, Newman CF et al. Kognitive Therapie der Sucht. Weinheim: Beltz, 1997
${ }^{36}$ Daley DC, Zukoff A. Improving treatment compliance: Counseling and systems strategies for substance abuse and dual disorders. Center City: Hazelden, 1999

${ }^{37}$ Walters ST, Ogle R, Martin JE. Perils and possibilities of group-based motivational interviewing. In: Miller WR, Rollnick S (Hrsg). Motivational interviewing. Preparing people for change. 2. Auflage. New York: Guilford Press, 2002; 377-390

${ }^{38}$ Alterman AI, Bedrick J, Howden D et al. Reducing waiting time for substance abuse treatment does not reduce attrition. Journal of Substance Abuse 1994; 6: 325-332

${ }^{39} \mathrm{He}$ H, Stark M, Fleming D et al. Facilitation into treatment or self-help among out-of-treatment IDUs in Portland: You can lead a horse to water, but ... Journal of Drug Issues. 1996; 26: 649-661

${ }^{40}$ Breitenbach G, Requardt H. Psychotherapie mit entmutigten Klienten. Heidelberg: Asanger, 2001

${ }^{41}$ Schu M, Schmid M, Görgen W et al. Case Management mit integriertem Motivational Interviewing. Manual. Köln/Frankfurt: FOGS/ISFF (unveröffentlicht)

42 Oliva H, Görgen W, Schlanstedt G et al. Case Management in der Suchtkranken- und Drogenhilfe. Abschlussbericht zum Kooperationsmodell nachgehende Sozialarbeit. Bd. 139 der Schriftenreihe des Bundesministeriums für Gesundheit. Baden-Baden: Nomos, 2001

${ }^{43}$ Schmid M, Vogt I. Case Management und motivierende Beratung. Suchttherapie 2001; 2: $73-79$

${ }^{44}$ Handmaker N, Packard M, Conforti K. Motivational Interviewing in the treatment of dual disorders. In: Miller WR, Rollnick S (Hrsg). Motivational interviewing. Preparing people for change. 2. Auflage. New York: Guilford Press, 2002; 362 - 376

45 Basdekis-Jozsa R, Krausz M. Komorbidität bei Suchterkrankungen. In: Backmund $\mathrm{M}$ (Hrsg). Ecomed-Medizin; 4. Ergänzungslieferung. Landsberg: ecomed Verlagsgesellschaft, 2003; 1-26

${ }^{46}$ Daley DC, Zukoff A. Improving compliance with the initial outpatient session among discharged inpatient dual diagnosis patients. Social Work 1998; 43: 470-473

47 Daley DC, Salloum IM, Zukoff A et al. Increasing treatment adherence among outpatients with depression and cocaine dependence: Results of a pilot study. American Journal of Psychiatry 1998; 155: 1611 - 1613

${ }^{48}$ Martino S, Caroll KM, O'Malley SS et al. Motivational interviewing with psychiatrically ill substance abusing patients. American Journal on Addictions 2000; 9: 88-91

${ }^{49}$ Carey K. Substance use reduction in the context of outpatient psychiatric treatment: A collaborative, motivational, harm reduction approach. Community Mental Health Journal 1996; 32: 291 - 306

50 Sciacca K. Removing barriers: Dual diagnosis treatment and motivational interviewing. Professional Counselor 1997; 12: 41-46

${ }^{51}$ Swanson AJ, Pantalon MV, Cohen KR. Motivational interviewing and treatment adherence among psychiatric and dually diagnosed patients. Journal of Nerveous and Mental Disease 1999; 187: 630-635

52 Barrowclough C. Cognitive behavioural intervention for clients with severe mental illness who have a substance misuse problem. Psychiatry and Rehabilitation Skills 2000; 42: 216-233

53 Barrowclough C, Haddock G, Tarrier N et al. Randomized controlled trail of motivational interviewing, cognitive behavior therapy, and family intervention for patients with comorbid schizophrenia and substance use disorders. American Journal of Psychiatry 2001; 158: $1706-1713$

${ }^{54}$ Kemp R, David A, Hayward P. Compliance therapy: An intervention targeting insight and treatment adherence in psychotic patients. Behavioural and Cognitive Psychotherapy 1996; 24: 331 - 350

${ }^{55} \mathrm{Kemp}$ R, Kirov G, Everitt B et al. Randomised controlled trial of compliance therapy: 18-month follow-up. British Journal of Psychiatry 1998; 172: $413-419$

${ }^{56}$ Ginsburg JID, Mann RE, Rotgers F et al. Motivational interviewing with Criminal Justice Populations. In: Miller WR, Rollnick S (Hrsg). Motivational interviewing. Preparing people for change. 2. Auflage. New York: Guilford Press, 2002: 333-346

${ }^{57}$ Baer JS, Peterson PL. Motivational interviewing with adolescents. In: Miller WR, Rollnick S (Hrsg). Motivational interviewing. Preparing people for change. 2. Auflage. New York: Guilford Press, 2002; $320-332$

${ }^{58}$ Hanewinkel R, Wiborg G. Riskantes Trinken im jungen Erwachsenenalter. Kiel: IFT-Nord, 2002 (Typoskript)

${ }^{59}$ Demmel R. Inflationäre Selbstwirksamkeitserwartungen rückfälliger Patienten: Selbstüberschätzung oder Zweckoptimismus? Sucht 2002; 48: $454-456$ 
${ }^{60}$ Dennis MI. The cannabis youth treatment (CYT) experiment: Preliminary findings. Rockville: Center for Substance Abuse Treatment, 2000

${ }^{61}$ Ingersoll KS, Wagner CC, Gharib S. Motivational groups for community substance abuse programs. Richmond: Mid-Atlantic Addiction Technology Transfer Center/Rockville: Center for Substance Abuse Treatment, 2000

62 Aubrey LL. Motivational interviewing with adolescents presenting for outpatient substance abuse treatment. Doctoral Dissertation: University of Mexico, 1998

${ }^{63}$ Stephens RS, Roffman RA, Curtin L. Comparison of extended versus brief treatment for Marijuana use. Journal of Consulting and Clinical Psychology 2000; 68: 898-908

${ }^{64}$ Miller WR, Sovereign RG, Krege B. Motivational interviewing with problem drinkers: II. The Drinker's Check-Up as a preventive intervention. Behavioural Psychotherapy 1988; 16: 251 -268

65 Dimeff LA, Baer JS, Kivlahan DR et al. Brief alcohol screening and intervention for college students (BASICS). New York: Guilford Press, 1999

${ }^{66}$ Strobl M, Klapper J, Pelzel KH et al. Suchthilfestatistik 2001 für Deutschland. Tabellenband für die ambulante Suchtkrankenhilfe. München: IFT im Internet: URL: http://www.ebis-ift.de (Stand: 1.3.2003)

${ }^{67}$ Schu M, Schlanstedt G, Oliva H. Hilfeplanung für chronisch mehrfachbeeinträchtigte Abhängige zwischen Anspruch und Wirklichkeit. Suchttherapie 2001; 2: $73-79$

${ }^{68}$ Rogers CR. Die nicht-direktive Beratung. München: Kindler, 1972

${ }^{69}$ Vogt I. Sozialarbeit mit Drogenabhängigen in einem „akzeptierenden“ Setting. In: Stöver H (Hrsg). Akzeptierende Drogenarbeit. Freiburg: Lambertus, 1999: 25-37

${ }^{70}$ Miller WR. Redescovering fire: Small interventions, large effects. Psychology of Addictive Behaviors 2000; 14: 6-18
${ }^{71}$ Project MATCH Research Group. Therapist effects in three treatments for alcohol problems. Psychotherapy Research 1998; 8: 455-474

72 Tossmann P, Nöcker G. Expertise zur strukturellen Weiterentwicklung der Suchtprävention in Berlin. Manuskript. Berlin: Delphi, 2003

${ }^{73}$ Schlömer H, Rodick A. Steigendem Cannabiskonsum begegnen - neue Erkenntnisse und Projekte der Schulbehörde zur Konsumvorbeugung und -reduzierung. Hamburg: Suchtpräventionsszentrum (unveröffentlichte Mitteilung des SPZ vom 21.6.2002)

${ }^{74}$ Perkonigg A. Patterns of cannabis use, abuse and dependence over time: incidence, progression and stability in a sample of 1228 adolescents. Addiction 1999; 94 (11): 1663 - 1678

75 Görgen W, Hartmann R, Oliva H. Frühintervention bei erstauffälligen Drogenkonsumenten - FreD. Ergebnisse der wissenschaftlichen Begleitung. Im Auftrag des Bundesministeriums für Gesundheit und Soziale Sicherung (BMGS). Köln: FOGS

${ }^{76}$ Marzinzik K, Gass J, Schmidt B. Motivierende Kurzinterventionen bei konsumierenden Jugendlichen. Prävention 24 (4) (in Vorbereitung)

${ }^{77}$ Schu M. Case Management in der Suchtkranken- und Drogenhilfe. In: Böllinger L, Stöver H (Hrsg). Drogenpraxis, Drogenrecht, Drogenpolitik - Handbuch für Drogenbenutzer, Eltern, Drogenberater, Ärzte und Juristen. Frankfurt: Fachhochschulverlag, 2002: 354-363

78 Baer R. Drogenhilfe zwischen Rausch und Nüchternheit. Suchttheorie, Drogenpolitik und Rehabilitationsalltag am Beispiel des Aebi-Hus/ Maison Blanche 1974-1999. Bern: Peter Lang, 2000

${ }^{79}$ Miller WR, Rollnick S. Motivierende Gesprächsführung. Ein Konzept zur Beratung von Menschen mit Suchtproblemen. Freiburg: Lambertus, 1999 